\title{
Reactive oxygen species, lipid peroxidation and enzymatic defence systems in human spermatozoa
}

\author{
J. F. Griveau ${ }^{1}$, E. Dumont ${ }^{2}$, P. Renard ${ }^{3}$, J. P. Callegari ${ }^{2}$ and \\ D. Le Lannou ${ }^{\mathrm{I}}$ \\ ${ }^{1}$ Cecos de l'Ouest, 1 bis rue de la cochardiere, 35000 Rennes, France; ${ }^{2}$ Profil, avenue du Général Leclerc, \\ Campus de beaulieu, 35700 Rennes, Erance; and ${ }^{3}$ Graf, 1 bis rue de la cochardiere, 35000 Rennes, France
}

\begin{abstract}
The reactive oxygen species, hydrogen peroxide $\left(\mathrm{H}_{2} \mathrm{O}_{2}\right)$ and superoxide anion $\left(\mathrm{O}_{2}{ }^{\circ}\right)$, were generated with a xanthine-xanthine oxidase system and their effect on human sperm function was studied. The action of reactive oxygen species on selected human spermatozoa resulted in a decreased capacity for ionophore-induced acrosome reaction, a decrease in sperm motility, an increase in the concentration of lipid hydroperoxides and a loss of membrane polyunsaturated fatty acids. $\mathrm{H}_{2} \mathrm{O}_{2}$ was the key intermediate of the deleterious effects exerted by the xanthine and xanthine oxidase. Among these parameters, the acrosome reaction appeared most susceptible to the reactive oxygen species generated by the xanthine-xanthine oxidase system, and was decreased without sperm motility being affected. Treatment with $\mathrm{H}_{2} \mathrm{O}_{2}$ was shown to inactivate several enzymatic activities involved in the antioxidant defence of spermatozoa : glutathione peroxidase, superoxide dismutase and glucose-6-phosphate dehydrogenase. $\mathrm{H}_{2} \mathrm{O}_{2}$ and $\mathrm{O}_{2}{ }^{\circ}-$ were shown to be involved in the lipid alterations triggered by the xanthine-xanthine oxidase system. Singlet oxygen is proposed to intervene in the lipoperoxidation process. The inefficacy of mannitol in protecting spermatozoa suggests that hydroxyl radicals were not produced in the extracellular medium.
\end{abstract}

\section{Introduction}

The role of reactive oxygen species in the pathophysiology of human sperm function has been emphasized in recent years (Jones et al., 1979; Alvarez et al., 1987; Aitken et al., 1989, 1991, 1993a, b; De Lamirande and Gagnon, 1992a, b). Their production in semen has been associated with loss of motility, decreased capacity for sperm-oocyte fusion and loss of fertility (Aitken et al., 1989, 1991).

In semen preparations, there are two major sources of reactive oxygen species: leucocytes (Aitken and West, 1990; Aitken et al., 1992a; Kessopoulou et al., 1992) and spermatozoa (Aitken and Clarkson, 1987; Alvarez et al., 1987; Aitken et al., 1992b; Iwasaki and Gagnon, 1992; Zini et al., 1993). Aitken and Clarkson (1987) proposed that production by spermatozoa was dependent upon a membrane-bound NADPH-oxidase. Hydrogen peroxide $\left(\mathrm{H}_{2} \mathrm{O}_{2}\right)$ produced by the dismutation of superoxide anion has been recognized as the most toxic oxidizing species for human spermatozoa (De Lamirande and Gagnon, 1992a; Aitken et al., 1993a). Owing to their high content of polyunsaturated fatty acids, it has been proposed that lipid peroxidation of the sperm plasma membrane is largely responsible for defective sperm function (Jones et al., 1979). Reactive oxygen species also affect the sperm axoneme as a result of ATP depletion (De Lamirande and Gagnon, 1992b), inhibit mitochondrial functions, and synthesis of DNA, RNA and

Received 2 May 1994. proteins (Comporti, 1989), produce cytoskeletal modifications (Hindshaw et al., 1986) and inhibit sperm-oocyte fusion (Aitken et al., 1993a).

Human spermatozoa possess enzymatic defence systems such as superoxide dismutase (SOD), glutathione peroxidasel reductase (GPX/GRD) and catalase (Nissen and Kreysel, 1983; Alvarez et al., 1987; Alvarez and Storey, 1989; Jeulin et al., 1989) to counteract the toxic effects induced by reactive oxygen species. Although correlations have been reported between the effectiveness of reactive oxygen species and the duration of sperm motility (Alvarez et al., 1987; Alvarez and Storey, 1989), the importance of their action has not been fully elucidated.

This study was designed to investigate the direct effects of reactive oxygen species on membrane phospholipids, enzymatic defence systems, ionophore-induced acrosome reaction and motility. Reactive oxygen species were produced using a combination of xanthine and xanthine oxidase, which catalyses the reduction of ground state oxygen to generate $\mathrm{O}_{2}{ }^{\circ-}$ and $\mathrm{H}_{2} \mathrm{O}_{2}$ by spontaneous dismutation of superoxide.

\section{Materials and Methods}

\section{Media and reagents}

$\mathrm{B}_{2}$ medium was obtained from Bio-Mérieux (Api system; Montalieu), Percoll from Pharmacia (Uppsala), Ham's F10 
medium from Flow Laboratories (Puteaux), $\mathrm{GB}_{24}$ from Theramex (Monaco) and rhodamine $B$ from Prolabo (Paris). Methanol, chloroform, acetic acid, heptane, dioxane, sulfuric acid and thin layer silica gel plates were obtained from Merck (Darmstadt). All other media and reagents were purchased from Sigma Chemical Company (St Louis, MO).

\section{Sperm preparation and induction of oxidative stress}

Semen samples were collected by masturbation from healthy donors or from men attending the fertility laboratory after a sexual abstinence of 2-3 days. Only spermatozoa exhibiting normal semen characteristics $\left(>20 \times 10^{6}\right.$ spermatozoa ml ${ }^{-1}$, $>50 \%$ motility and $>50 \%$ normal forms) were used. After $15 \mathrm{~min}$ liquefaction at $37^{\circ} \mathrm{C}$, spermatozoa were fractionated on a four-step discontinuous Percoll gradient (46-69-83-92\%). Percoll was made isotonic by adding 0.8 volumes of $0.4 \mathrm{~mol}$ Hepes-buffered Ham's F10 $\mathrm{I}^{-1}(\mathrm{pH} 7.4)$ to 9.2 volumes of Percoll. The resulting solution was further diluted with $0.04 \mathrm{~mol}$ Hepes-buffered Ham's F10 $1^{-1}$ to give 46,69 and $83 \%$ Percoll solutions. Semen was layered on the top of the gradient and centrifuged at $500 \mathrm{~g}$ for $20 \mathrm{~min}$ at room temperature. Spermatozoa from the $92 \%$ Percoll fractions were resuspended in $5 \mathrm{ml}$ of $\mathrm{B}_{2}$ medium and centrifuged at $500 \mathrm{~g}$ for $5 \mathrm{~min}$ at room temperature. The supernatant was discarded and spermatozoa were resuspended at a concentration of $20 \times 10^{6}$ spermatozoa $\mathrm{ml}^{-1}$ in $\mathrm{B}_{2}$ medium.

Spermatozoa were then incubated in $\mathrm{B}_{2}$ medium at $37^{\circ} \mathrm{C}$ under a $5 \% \mathrm{CO}_{2}$ atmosphere to allow capacitation with a combination of xanthine ( $\mathrm{X}$ : $100-200 \mu \mathrm{mol} \mathrm{1^{-1 }}$ ) and xanthine oxidase (XO: $\left.1-50 \mathrm{mU} \mathrm{ml}^{-1}\right)$, or with $\mathrm{H}_{2} \mathrm{O}_{2}\left(500 \mu \mathrm{mol} \mathrm{I}^{-1}\right)$ or mercaptosuccinate (MCS: $200 \mu \mathrm{mol} \mathrm{l}^{-1}$ ). The reactive oxygen species scavengers: superoxide dismutase $\left(500 \mathrm{U} \mathrm{ml}^{-1}\right)$ catalase $\left(500 \mathrm{U} \mathrm{ml}^{-1}\right)$, mannitol $\left(100 \mu \mathrm{mol} \mathrm{l}^{-1}\right)$ and histidine (10 mmol $\mathrm{l}^{-1}$ ) were also tested.

\section{Analysis of sperm motility}

Sperm motility was analysed using the ATS 40 motility analyser (J. C. Diffusion International, La Ferte Fresnel) in a $20 \mu \mathrm{mol} 1^{-1}$ depth chamber (Fertility Technology Inc., Natick, $\mathrm{MA}$ ) at $37^{\circ} \mathrm{C}$. This system was previously validated for measuring the specific movement parameters of human spermatozoa (Le Lannou et al., 1992). System parameter settings for this analysis were 40 frames $\mathrm{s}^{-1}$ recorded, 30 consecutive frames analysed, spermatozoon minimum size 8 pixels, spermatozoon maximum size 20 pixels, $20 \mu \mathrm{m} \mathrm{s}^{-1}$ threshold velocity and $300 \mu \mathrm{m} \mathrm{s}^{-1}$ maximum velocity.

A minimum of 100 trajectories were analysed for each sample. The characteristics of movement recorded were motility (\%), curvilinear velocity (VCL, $\mu \mathrm{m} \mathrm{s}^{-1}$ ), straight line velocity (VSL, $\mu \mathrm{m} \mathrm{s}^{-1}$ ), amplitude of lateral head displacement $(\mathrm{ALH}, \mu \mathrm{m})$, linearity (LIN, \%) and percentage of hyperactivated spermatozoa (percentage of cells exhibiting a VCL $>100 \mu \mathrm{m}$ $\mathrm{s}^{-1}$, LIN $<40 \%$ and ALH $>4.5 \mu \mathrm{m}$; adapted from Mortimer and Mortimer, 1990; Burkman, 1991).

\section{Induction and evaluation of acrosome reaction}

Acrosome reaction was evaluated after $6 \mathrm{~h}$ of incubation, when it was chemically induced with $10 \mu \mathrm{mol} A 23187 \mathrm{l}^{-1}$ (stored at $10^{-3} \mathrm{~mol} \mathrm{l}^{-1}$ in ethanol at $-20^{\circ} \mathrm{C}$ before use) in $\mathrm{B}_{2}$ medium for $1 \mathrm{~h}$ at $37^{\circ} \mathrm{C}$ and in an atmosphere of $5 \% \mathrm{CO}_{2}$ and $95 \%$ air. Five minutes before the end of the incubation, the vital stain propidium iodide $\left(10^{-9} \mathrm{~mol} \mathrm{l}^{-1}\right.$ final) was added to the sperm suspension. After washing by centrifugation at $500 \mathrm{~g}$ for $5 \mathrm{~min}$ in PBS with $0.1 \% \mathrm{BSA}$, the spermatozoa were resuspended with $\mathrm{GB}_{24}$ antibody ( $1 \mu \mathrm{g}$ : $100 \mu \mathrm{l} \mathrm{PBS}$ ), a mouse monoclonal antibody that recognizes an inner acrosomal membrane-associated protein (Fenichel et al., 1990). After incubation for $45 \mathrm{~min}$ at room temperature, the spermatozoa were washed by centrifugation at $500 \mathrm{~g}$ for $5 \mathrm{~min}$ with PBS-BSA and resuspended with FITC-conjugated anti-mouse immunoglobulin at 1:50 in PBS-BSA for $30 \mathrm{~min}$. Spermatozoa were then washed by centrifugation at $500 \mathrm{~g}$ for $5 \mathrm{~min}$ with PBS-BSA and immediately observed by fluorescence microscopy. Two-hundred spermatozoa were evaluated for each sample. Only spermatozoa devoid of red fluorescence (given by propidium iodide) and exhibiting a green homogeneous fluorescence pattern distributed over the entire acrosome cap were scored as positive (living acrosome-reacted spermatozoa). The results were expressed as percentages of positive spermatozoa.

\section{Assay of reactive oxygen species}

The generation of $\mathrm{O}_{2}{ }^{\mathrm{O}-}$ by the combination of xanthine $\left(200 \mu \mathrm{mol} \mathrm{l}^{-1}\right)$ and xanthine oxidase $\left(50 \mathrm{mU} \mathrm{ml}^{-1}\right)$ was monitored at $550 \mathrm{~nm}$ by the reduction of ferricytochrome $C$ (Gavella et al., 1991). The specificity of the assay was assessed by adding $500 \mathrm{U}$ superoxide dismutase into the control incubation.

The production of $\mathrm{H}_{2} \mathrm{O}_{2}$ in the same system was measured at $510 \mathrm{~nm}$ using the peroxidase-catalysed oxidation of 4-aminoantipyrine in the presence of phenol (Aitken et al., 1993a). The specificity of the assay was assured by adding $500 \mathrm{U}$ catalase into the control incubation.

\section{Assay of superoxide dismutase-like activity}

Superoxide dismutase-like activity was measured by the inhibition of the ferricytochrome $\mathrm{C}$ reduction due to the $\mathrm{O}_{2}{ }^{\mathrm{O}}$ generated by $200 \mu \mathrm{mol}$ xanthine $\mathrm{l}^{-1}$ and $10 \mathrm{mU}$ xanthine oxidase (Alvarez and Storey, 1983).

The extraction procedure was that of Kobayashi et al. (1991). After $3 \mathrm{~h}$ of treatment, excess xanthine-xanthine oxidase, $\mathrm{H}_{2} \mathrm{O}_{2}$ or mercaptosuccinate was removed by centrifugation $(500 \mathrm{~g}, 5 \mathrm{~min})$, and spermatozoa were treated for $10 \mathrm{~min}$ with $10 \mathrm{mmol}$ potassium phosphate buffer $\mathrm{l}^{-1}$. Complete disruption of spermatozoa was achieved by sonicating them for 2 min at $4^{\circ} \mathrm{C}$ in $20 \mathrm{~s}$ bursts with $20 \mathrm{~s}$ intervals between bursts to avoid heating. The supernatant was then collected by centrifugation for $10 \mathrm{~min}$ at $1800 \mathrm{~g}$ and $4^{\circ} \mathrm{C}$, and used for the measurement of superoxide dismutase-like activity. The reaction was initiated by the addition of $\mathrm{XO}$ and monitored at $25^{\circ} \mathrm{C}$. One unit of activity was defined as the amount of enzyme required to inhibit the change of absorbance at $550 \mathrm{~nm}$ by $50 \%$. 
Assay of glutathione peroxidase and glutathione reductase activities and glutathione content

The activities of glutathione peroxidase and glutathione reductase and glutathione content were determined in hypoosmotically treated spermatozoa after $3 \mathrm{~h}$ of incubation with xanthine-xanthine oxidase, $\mathrm{H}_{2} \mathrm{O}_{2}$ or mercaptosuccinate and based on the coupled oxidation of NADPH, and reduction of oxidized glutathione (GSSG) by glutathione reductase (GRD) (Alvarez et al., 1987). In this series of experiments, enzymatic activities and glutathione content were determined in the whole hypotonic sperm suspension. The change in absorbance due to the oxidation of NADPH was monitored at $365 \mathrm{~nm}$.

\section{Assay of glucose-6-phosphate dehydrogenase activity}

Glucose-6-phosphate dehydrogenase (G6PDH) activity was measured using a kit (Sigma Diagnostics, St Louis, MO) after $3 \mathrm{~h}$ of incubation with xanthine $\left(200 \mu \mathrm{mol} \mathrm{l}^{-1}\right)$ and xanthine oxidase $\left(50 \mathrm{mU} \mathrm{ml}^{-1}\right)$. The increase in absorbance due to the formation of NADPH (proportional to the G6PDH activity) was recorded using a spectrophotometer at $340 \mathrm{~nm}$. The reduction of $\mathrm{NADP}^{+}$was linear for up to $20 \mathrm{~min}$.

\section{Extraction and analysis of sperm phospholipid}

Semen from 2-3 donors was pooled to obtain sufficient phospholipids, and spermatozoa were selected on Percoll gradient and treated with xanthine $\left(200 \mu \mathrm{mol} \mathrm{l}^{-1}\right)$ and xanthine oxidase $\left(50 \mathrm{mU} \mathrm{ml}^{-1}\right)$. After incubation for $6 \mathrm{~h}$, the lipids were extracted with chloroform/methanol $(2 / 1, v / v)$ according to the method of Folch et al. (1957). The mixture of lipids was fractionated by thin layer chromatography (TLC) using the solvent system hexane/diethylether/acetic acid (90/10/0.5, $\mathrm{v} / \mathrm{v} / \mathrm{v}$ ) for $45 \mathrm{~min}$ at room temperature. The different spots of lipids were visualized in ultraviolet after reaction with rhodamine and identified by comparison with lipid standards. The silica gel was scraped and the fatty acids transesterified according to the method of Hagenfeld (1966). Fatty acid methyl esters were then analysed by gas liquid chromatography (GLC) on a Chrompack CP 9001 apparatus equipped with a BPX70 capillary column (SGE, villeneuve-Saint Georges), $25 \mathrm{~m} \times 0.32 \mathrm{~mm}$ i.d., film thickness $0.22 \mu \mathrm{mol} \mathrm{l}^{-1}$ and a flame ionization detector. The vector gas was nitrogen. The analysis of fatty acids was carried out with an internal standard, nonadecanoic acid (19:0). The results obtained are given as percentages of the total fatty acids.

\section{Lipid hydroperoxide determination}

Amounts of lipid hydroperoxides were spectrophotometrically determined after incubation for $3 \mathrm{~h}$ according to the method developed by Hicks and Gebicki (1979) and based on the liberation of iodine after the 1:I stoichiometric reaction between hydroperoxides and potassium iodide. Briefly, total lipid extracts were prepared from spermatozoa as described above and stored under liquid nitrogen. Twentyfive microlitres of the extract were added to $1.8 \mathrm{ml}$ deoxygenated methanol/acetic acid $(1 / 1, \mathrm{v} / \mathrm{v})$ and $1.9 \mathrm{ml}$ of a $20 \%$ $(\mathrm{w} / \mathrm{v})$ potassium iodide solution in methanol. The change in absorbance was recorded at $360 \mathrm{~nm}$ until any further change was small and linear with time. Amounts of lipid hydroperoxides were calculated using the extinction coefficient of $2.80 \times 10^{4} \mathrm{I} \mathrm{mol}^{-1} \mathrm{~cm}^{-1}$.

\section{Singlet oxygen determination}

Singlet oxygen formation was investigated by the use of 1,3-diphenylisobenzofuran (DPBF), which forms 2,2 odibenzoylbenzene (DBB) when it reacts with ${ }^{1} \mathrm{O}_{2}$ (Pederson and Aust, 1973). DPBF (10 mmol $\mathrm{l}^{-1}$ ) was first dissolved in chloroform, and the solvent was removed under a stream of $\mathrm{N}_{2}$ and the deposit was emulsified in PBS by sonication. The reaction was initiated by addition of xanthine $\left(200 \mu \mathrm{mol} \mathrm{l^{-1 }}\right)$ and xanthine oxidase $\left(50 \mathrm{mU} \mathrm{ml}^{-1}\right)$. The reaction mixture was incubated for $1 \mathrm{~h}$ at $37^{\circ} \mathrm{C}$ and extracted with $0.5 \mathrm{ml}$ chloroform. One-hundred microlitres of each extract was applied to thin layer plate silica gel and developed by heptane/dioxane $(3 / 1, v / v)$. The plate was then sprayed with $0.5 \%(w / v)$ of 2.4 dinitrophenylhydrazine in $2 \mathrm{~mol} \mathrm{HCl}^{-1}$.

\section{Statistical analysis}

Unless stated otherwise, the statistical significance of the results was assessed using the paired Student's $t$ test using the STATWORKS statistical package (Heyden and Sons Inc., Philadelphia, PA). Differences with values of $P<0.05$ were considered to be statistically significant.

\section{Results}

Production of reactive oxygen species and effects on sperm motility and acrosome reaction

A combination of xanthine $\left(200 \mu \mathrm{mol} \mathrm{1^{-1 }}\right)$ and xanthine oxidase $\left(50 \mathrm{mU} \mathrm{ml} \mathrm{m}^{-1}\right)$ resulted in the production of $40.0 \pm 0.7 \mu \mathrm{mol} \mathrm{O}{ }_{2}^{\circ-} \mathrm{I}^{-1}$ and $79.0 \pm 3.2 \mu \mathrm{mol} \mathrm{H}_{2} \mathrm{O}_{2} \mathrm{I}^{-1}$ (from the spontaneous dismutation of $\mathrm{O}_{2}{ }^{\mathrm{o}-}$ ) over 4 and $6 \mathrm{~min}$, respectively (Fig. 1). The specificity of the assays was assured by superoxide dismutase and catalase which respectively scavenged $\mathrm{O}_{2}{ }^{\circ-}$ and $\mathrm{H}_{2} \mathrm{O}_{2}$. Within $5 \mathrm{~min}, \mathrm{O}_{2}{ }^{\circ-}$ could not be detected in the medium, whereas $\mathrm{H}_{2} \mathrm{O}_{2}$ decreased only slightly for several hours. However, in the presence of spermatozoa $\left(20 \times 10^{6}\right.$ spermatozoa ml $\left.{ }^{-1}\right)$, the amount of $\mathrm{H}_{2} \mathrm{O}_{2}$ decreased very rapidly and was insignificant after $1 \mathrm{~h}$ of incubation (data not shown).

The response of spermatozoa to this profile of reactive oxygen species production was a progressive and significant decrease with time in percentage motility $(P<0.01)$ and every parameter of sperm movement (Table 1) except for ALH, which was even significantly increased $(P<0.05)$ after incubation for $3 \mathrm{~h}$.

The influence of reactive oxygen species on the capacity of human spermatozoa to undergo the acrosome reaction after capacitation for $6 \mathrm{~h}$ was assessed with a concentration of xanthine at which the percentage of motile spermatozoa was unaltered. Exposure to $100 \mu \mathrm{mol}$ xanthine $1^{-1}$ and $50 \mathrm{mU}$ 


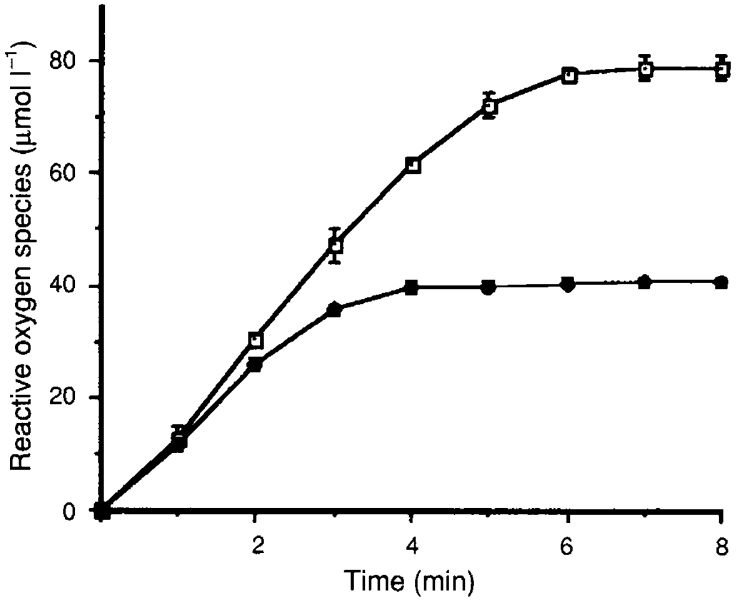

Fig. 1. Time course of reactive oxygen species production induced by $200 \mu \mathrm{mol}$ xanthine $\mathrm{l}^{-1}$ and $50 \mathrm{mU}$ xanthine oxidase $\mathrm{ml}^{-1}$. The generation of $\mathrm{O}_{2}{ }^{\mathrm{O}-}$ was monitored by the reduction of ferricytochrome $\mathrm{C}$, and the generation of $\mathrm{H}_{2} \mathrm{O}_{2}$ was monitored by the peroxidase-catalysed oxidation of 4-aminoantipyrine in the presence of phenol. Values are means $\pm \mathrm{SEM}, n=3 . \sqsupset: \mathrm{H}_{2} \mathrm{O}_{2} ; \mathbf{\square}: \mathrm{O}_{2}{ }^{\circ-}$.

xanthine oxidase $\mathrm{ml}^{-1}$ was compatible with the maintenance of the percentage of motility for up to $6 \mathrm{~h}(85.0 \pm 4.0 \%$ for control versus $77.8 \pm 4.7 \%$ for xanthine-xanthine oxidase). Despite an unchanged sperm motility, the percentage of the ionophore-induced acrosome reaction was significantly diminished $(26.2 \pm 3.1 \%$ for control versus $4.5 \pm 1.5 \%$ for xanthine-xanthine oxidase, $P<0.001$ ).

A series of experiments was performed in which the concentration of xanthine was fixed at $100 \mu \mathrm{mol} \mathrm{l}^{-1}$ but that of xanthine oxidase varied from 1 to $50 \mathrm{mU} \mathrm{ml}^{-1}$ (Fig. 2) to determine whether the speed at which reactive oxygen species were generated had any influence on the ionophore-induced acrosome reaction. As the concentration of enzyme increased, the effects on the ionophore-induced acrosome reaction were more pronounced. With the lowest concentration of xanthine oxidase $\left(1 \mathrm{mU} \mathrm{ml}^{-1}\right)$, the response to $\mathrm{A} 23187$ was unchanged, whereas at $5 \mathrm{mU} \mathrm{ml}^{-1}$ or above, treated spermatozoa exhibited a dose-dependent diminished response to induction of the acrosome reaction.
Influence of the xanthine oxidase system on sperm phospholipids

An oxidation of the membrane fatty acids was observed after exposure of the human spermatozoa to xanthine $\left(200 \mu \mathrm{mol} \mathrm{l}^{-1}\right)$ and xanthine oxidase $\left(50 \mathrm{mU} \mathrm{ml}^{-1}\right)$. GLC analysis revealed that the polyunsaturated fatty acid (PUFA) concentrations were preferentially affected (Fig. 3a) and dropped by $20 \%$, whereas saturated and mono-unsaturated fatty acid concentrations remained stable.

A more detailed analysis of the behaviour of the major phospholipid PUFAs revealed that they were differentially affected (Fig. 3b). Linoleic acid (18:2n-6) showed a high resistance to reactive oxygen species exposure and remained stable at $98.6 \pm 3.2 \%$ of its initial value. The two other major PUFAs: arachidonic acid (20:4n-6) and docosaexaenoic acid $(22: 6 \mathrm{n}-3)$ were more affected with, respectively, $75.0 \pm 12.9 \%$ and $72.3 \pm 5.6 \%$ of their initial concentrations remaining after incubation for $6 \mathrm{~h}$.

\section{Effects of reactive oxygen species scavengers}

A series of specific scavengers was tested on spermatozoa exposed to xanthine $\left(200 \mu \mathrm{mol} \mathrm{l} \mathrm{l}^{-1}\right)$ and xanthine oxidase $\left(50 \mathrm{mU} \mathrm{ml}{ }^{-1}\right)$ to determine which reactive oxygen species was responsible for the decreases in motility, acrosome reaction and PUFA content.

Among the scavengers tested, only catalase completely reversed the toxic effects induced by the xanthine-xanthine oxidase system on all the parameters considered (Table 2).

Superoxide dismutase prevented loss of PUFA content, with $96.5 \pm 4.9 \%$ of initial concentrations remaining after $6 \mathrm{~h}$ of incubation (arachidonic and docosahexaenoic acids were equally protected: $96.8 \pm 1.3$ and $96.0 \pm 1.4 \%$ of native spermatozoa, respectively) but exhibited no capacity to preserve sperm motility and ionophore-induced acrosome reaction.

The hydroxyl radical scavenger, mannitol $\left(100 \mathrm{mmol} \mathrm{l}^{-1}\right)$, did not modify any of the cytotoxic effects of the reactive oxygen species.

\section{Reactive oxygen species, enzymatic defence systems and lipid hydroperoxides}

Treatment of spermatozoa with the combination of xanthine $\left(200 \mu \mathrm{mol} \mathrm{l}^{-1}\right)$ and xanthine oxidase $\left(50 \mathrm{mU} \mathrm{ml}^{-1}\right)$ caused a

Table 1. Influence of the combination of xanthine $\left(200 \mu \mathrm{mol} \mathrm{l}^{-1}\right)$ and xanthine oxidase $\left(50 \mathrm{mU} \mathrm{ml}^{-1}\right)$ on the movement characteristics of human spermatozoa after 3 and $6 \mathrm{~h}$ of incubation

\begin{tabular}{|c|c|c|c|c|}
\hline \multirow[b]{3}{*}{ Movement characteristics } & \multirow{2}{*}{\multicolumn{2}{|c|}{$3 \mathrm{~h}$}} & \multirow{2}{*}{\multicolumn{2}{|c|}{$6 \mathrm{~h}$}} \\
\hline & & & & \\
\hline & Control & $x-X O$ & Control & $X-X O$ \\
\hline Motility (\%) & $87.7 \pm 2.3$ & $63.7 \pm 8.8^{*}$ & $85.7 \pm 4.1$ & $54.2 \pm 2.9^{*}$ \\
\hline$V C L\left(\mu \mathrm{m} \mathrm{s}^{-1}\right)$ & $92.0 \pm 2.3$ & $75.4 \pm 2.1^{* * *}$ & $90.8 \pm 2.6$ & $66.6 \pm 2.9^{* * *}$ \\
\hline $\operatorname{VSL}\left(\mu \mathrm{m} \mathrm{s}^{-1}\right)$ & $69.7 \pm 5.6$ & $47.7 \pm 2.6^{* *}$ & $70.1 \pm 3.1$ & $43.8 \pm 2.8^{* * *}$ \\
\hline $\operatorname{LIN}(\%)$ & $75.2 \pm 4.4$ & $62.9 \pm 2.5^{*}$ & $76.5 \pm 1.7$ & $64.3 \pm 2.3^{* *}$ \\
\hline $\operatorname{ALH}\left(\mu \mathrm{m} \mathrm{s}^{-1}\right)$ & $3.2 \pm 0.3$ & $4.0 \pm 0.2^{*}$ & $3.2 \pm 0.2$ & $3.3 \pm 0.2$ \\
\hline Hyperactivated (\%) & $10.2 \pm 3.1$ & $1.6 \pm 1.0^{* * *}$ & $3.7 \pm 2.3$ & $1.6 \pm 1.1$ \\
\hline
\end{tabular}

Values are means \pm SEM, $n=10 .{ }^{*} P<0.05 ; * *<0.01 ; * * P<0.001$ compared with control values.

VCL: curvilinear velocity; VSL: straight line velocity; LIN: linearity; ALH: lateral head displacement. 


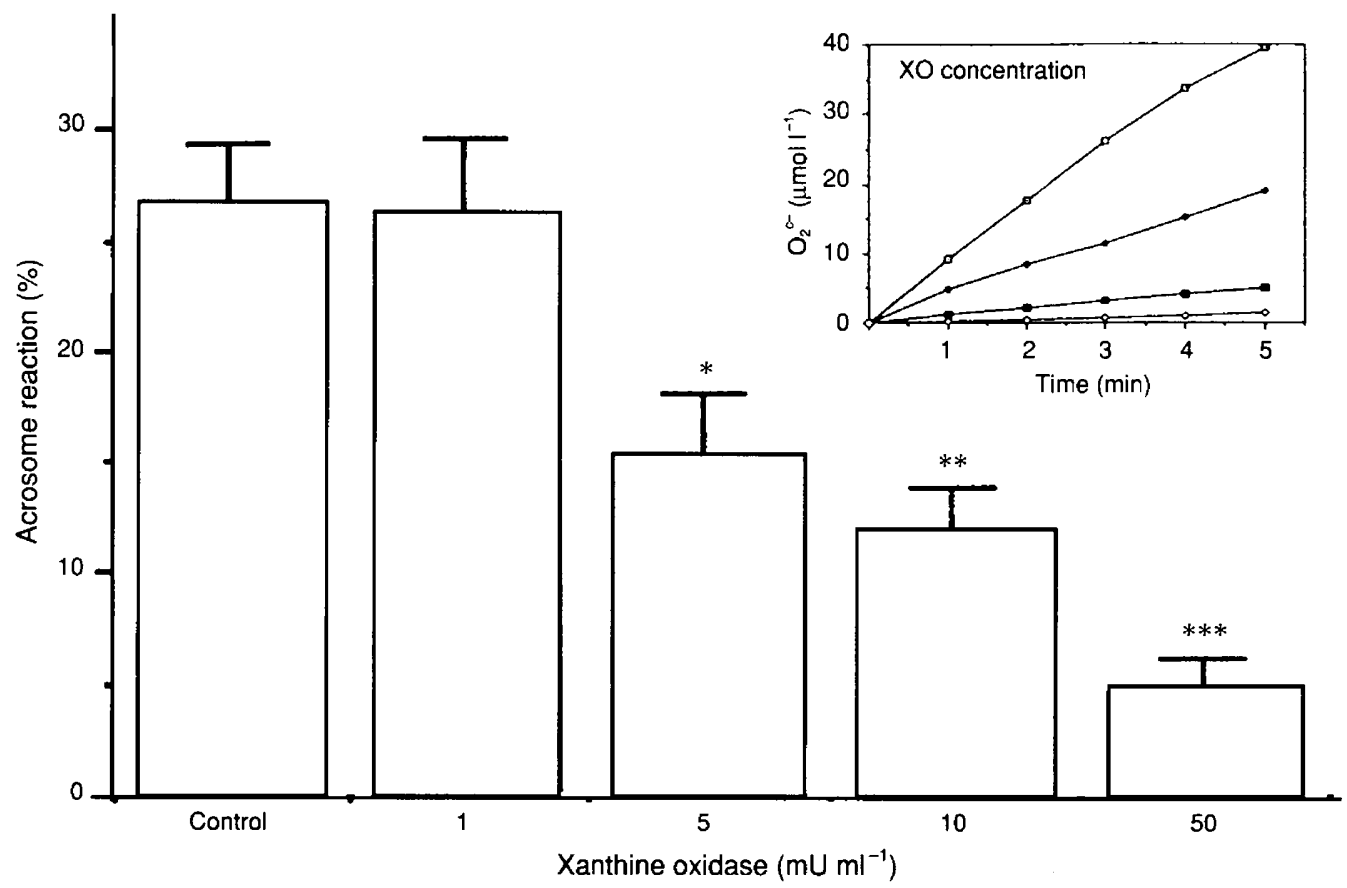

Fig. 2. Effects of various concentrations of xanthine oxidase on the capacity of human spermatozoa to undergo the acrosome reaction after exposure to $100 \mu \mathrm{mol}$ xanthine $\mathrm{l}^{-1}$. Acrosome reaction was induced after $6 \mathrm{~h}$ of capacitation by $10 \mu \mathrm{mol}$ calcium ionophore $\mathrm{A} 23187 \mathrm{l}^{-1}$. Values are means $\pm \operatorname{SEM}, n=5$. ${ }^{*} P<0.05 ; * * P<0.01$; ***P $P 0.001$ compared with control values. : $50 \mathrm{mU} \mathrm{ml}^{-1}$; :: $20 \mathrm{mU} \mathrm{ml}^{-1}$; :: $5 \mathrm{mU} \mathrm{ml}^{-1}$; : $1 \mathrm{mU} \mathrm{ml}^{-1}$.

significant decrease in superoxide dismutase-like and glutathione peroxidase activities (Table 3). In contrast, glutathione reductase activity was not affected by this treatment. A concomitant oxidation of reduced glutathione (GSH) to oxidised glutathione (GSSG) and a rise in the concentration of lipid hydroperoxides was observed.

Catalase provided protection against inhibition of both superoxide dismutase-like and glutathione peroxidase activities. Oxidation of GSH and an increase in lipid hydroperoxides were also prevented.

Although superoxide dismutase offered a partial protection against the rise in concentration of lipid hydroperoxides, its presence in the medium of incubation failed to protect glutathione peroxidase activity.

Treatment with $500 \mu \mathrm{mol} \mathrm{H}_{2} \mathrm{O}_{2} \mathrm{l}^{-1}$ led to the same decrease in superoxide dismutase-like and glutathione peroxidase activities, GSH oxidation and rise in concentration of lipid hydroperoxides as that observed with the xanthine-xanthine oxidase system.

The direct inactivation of glutathione peroxidase by $200 \mu \mathrm{mol}$ mercaptosuccinate $1^{-1}$ (a specific inhibitor of glutathione peroxidase) emphasized the important role played by this enzymatic system in protection against oxidative stress. Although superoxide dismutase-like activity was maintained and a complete conversion of GSSG to GSH observed, concentrations of lipid hydroperoxides were significantly $(P<0.01)$ increased.

The accumulation of GSSG, despite normal glutathione reductase activity, led us to investigate the production of NADPH (which allows the recycling of GSSG to GSH by glutathione reductase) by G6PDH, an enzyme of the pentose phosphate cycle (the main metabolic pathway leading to the production of NADPH in the cell). In agreement with our hypothesis, treatment of spermatozoa with the combination of xanthine $\left(200 \mu \mathrm{mol} \mathrm{l}^{-1}\right)$ and xanthine oxidase $\left(50 \mathrm{mU} \mathrm{ml}^{-1}\right)$ resulted in a significant decrease $(P<0.005)$ in the production of NADPH by the G6PDH $(0.98 \pm 0.02$ versus $0.74 \pm$ $0.05 \mu \mathrm{mol}$ NADPH formed $\min ^{-1} 10^{-8}$ spermatozoa, $n=6$ ).

\section{Effects of singlet oxygen}

In the study reported here, we have shown that external superoxide dismutase protected the spermatozoa PUFAs against oxidation after incubation with the xanthine-xanthine oxidase system. Some previous studies suggest that the dismutation of lipid peroxyl radicals (Nakano et al., 1976) and the spontaneous dismutation of $\mathrm{O}_{2}{ }^{-}$in aqueous solution (Corey et al., 1987) produce ${ }^{1} \mathrm{O}_{2}$. It has also already been shown that ${ }^{1} \mathrm{O}_{2}$ may be produced by xanthine oxidase (Kellogg and Fridovich, 1975, 1977).

The generation of ${ }^{1} \mathrm{O}_{2}$ in the pro-oxidant system used in this study was verified by investigating the formation of $o$-dibenzoylbenzene, by reaction of ${ }^{1} \mathrm{O}_{2}$ with 1,3 diphenylisobenzofuran by thin layer chromatography. The aerobic incubation of $200 \mu \mathrm{mol}$ xanthine $1^{-1}$ and $50 \mathrm{mU}$ xanthine oxidase $\mathrm{ml}^{-1}$ converted 1,3 diphenylisobenzofuran to o-dibenzoylbenzene (data not shown). This reaction was inhibited by superoxidase dismutase.

The role played by ${ }^{1} \mathrm{O}_{2}$ in the alterations induced by the xanthine-xanthine oxidase system was studied with histidine, a ${ }^{1} \mathrm{O}_{2}$ scavenger. It was first verified that histidine did not interfere with the production of reactive oxygen species by xanthine and xanthine oxidase. Histidine partially prevented 

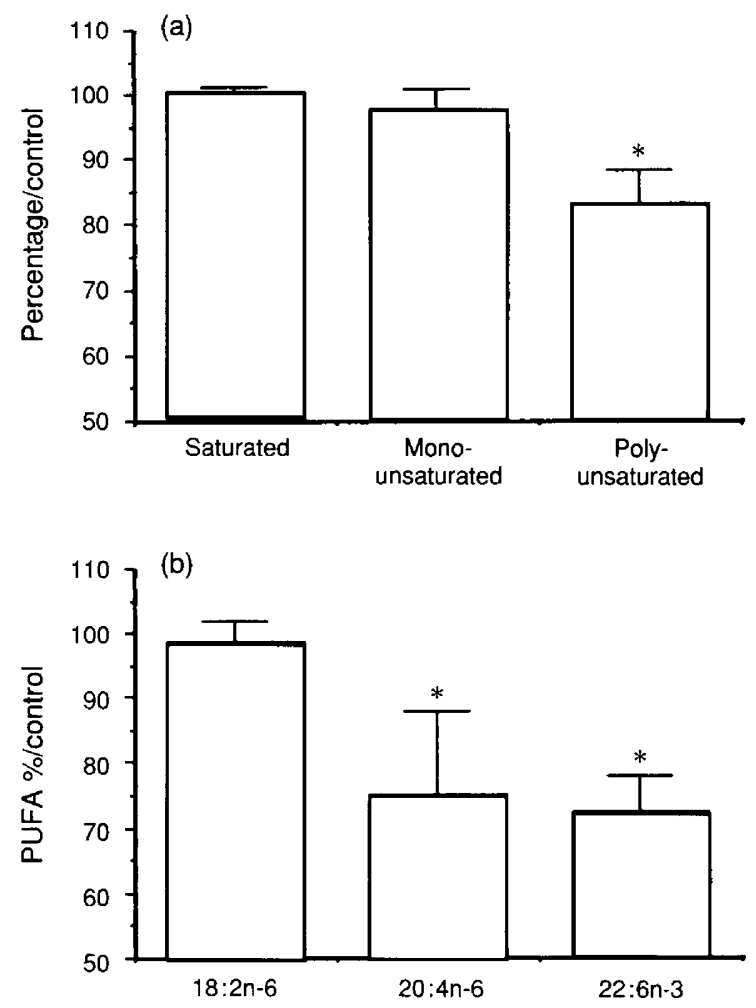

Fig. 3. Gas liquid chromatography analysis of the phospholipid fatty acid content after $6 \mathrm{~h}$ exposure to $200 \mu \mathrm{mol}$ xanthine $\mathrm{l}^{-1}$ and $50 \mathrm{mU}$ xanthine oxidase $\mathrm{ml}^{-1}$. (a) Relative modifications of the proportions of the different classes of fatty acids. Results are expressed as percentages of intact fatty acids remaining compared with the control, considered to be $100 \%$ for each class. Values are means $\pm \mathrm{SEM}, n=5$. ${ }^{*} P<0.01$ compared with the control. (b) Analysis of the main phospholipid polyunsaturated fatty acids, linoleic acid (18:2 n-6), arachidonic acid (20:4 n-6) and docosahexaenoic acid (22:6 n-3). Results are expressed as percentages of intact polyunsaturated fatty acid (PUFA) compared with the control. Values are means $\pm \mathrm{SEM}, n=5$. $* P<0.001$ compared with the control.

the increase in concentration of lipid hydroperoxides and the loss of PUFA content in sperm plasma phospholipids (Table 4).

\section{Discussion}

The xanthine-xanthine oxidase system is known to produce reactive oxygen species that are involved in cellular degradation in several cell types, including spermatozoa. The present study shows that exposure of selected human spermatozoa to the reactive oxygen species generated by a xanthine-xanthine oxidase system leads to an increase of lipid hydroperoxide content, loss of membrane PUFAs, diminished capacity to undergo the acrosomal reaction and loss of motility. The ionophore-induced acrosome reaction was a more sensitive parameter than motility in relation to oxidative stress, and both the rate of production and the amount of reactive oxygen species generated by the xanthine-xanthine oxidase system influenced the inhibition of the acrosome reaction. The use of specific scavengers (catalase, superoxide dismutase) allowed us to show that $\mathrm{H}_{2} \mathrm{O}_{2}$ and $\mathrm{O}_{2}{ }^{\circ-}$ play a role in the alterations induced by xanthine and xanthine oxidase. The presence of catalase completely reversed all the cytotoxic effects observed, whereas superoxide dismutase inhibited only the increase of lipid hydroperoxide content and the degradation of PUFAs, but did not have any protective effect on the ionophoreinduced acrosome reaction and motility. In fact, all the effects of the xanthine-xanthine oxidase system on the enzymatic antioxidant defence systems, glutathione and lipid hydroperoxide concentrations can be mimicked by the direct addition of $\mathrm{H}_{2} \mathrm{O}_{2}$, and can be inhibited by the presence of catalase. These results are in agreement with previous studies in which $\mathrm{H}_{2} \mathrm{O}_{2}$ was proposed as the most toxic reactive oxygen species for human spermatozoa (Alvarez and Storey, 1989; De Lamirande and Gagnon, 1992a; Aitken et al., 1993a).

The accumulation of GSSG after an incubation of spermatozoa with $\mathrm{H}_{2} \mathrm{O}_{2}$ was described by Alvarez and Storey (1989) who concluded that this accumulation was responsible for a decrease in glutathione peroxidase activity. The results presented here show that glutathione peroxidase is inhibited per se and not by a lack of its substrate GSH after exposure to $\mathrm{H}_{2} \mathrm{O}_{2}$ or the xanthine-xanthine oxidase system. They also show that GSSG accumulates in spermatozoa in spite of a decreased glutathione peroxidase activity and an unchanged glutathione reductase activity. The efficacy of the protection against lipoperoxidation afforded by glutathione peroxidase and GSH is dependent upon the recycling of oxidized glutathione (GSSG) into its reduced form (GSH). This recycling is done by glutathione reductase and needs NADPH as a reducing cofactor. The accumulation of GSSG despite normal glutathione reductase activity may be linked to a lack of NADPH in the spermatozoa. The main metabolic pathway leading to the production of NADPH in cells is the pentose phosphate cycle in which G6PDH transforms glucose-6-phosphate into phospho-6-glucono-lactone with the production of NADPH.

Table 2. Effects of reactive oxygen species scavengers on motility, ionophore-induced acrosome reaction and membrane polyunsaturated fatty acid (PUFA) content after $6 \mathrm{~h}$ of incubation with the combination of xanthine $(X)\left(200 \mu \mathrm{mol} \mathrm{I}^{-1}\right)$ and xanthine oxidase $(\mathrm{XO})\left(50 \mathrm{mU} \mathrm{ml}^{-1}\right)$

\begin{tabular}{lccccc}
\hline Parameter & Control & $X-X O$ & $X-X O+$ catalase & $X-X O+$ superoxide dismutase & $X-X O+$ mannitol \\
\hline Motility (\%) & $87.6 \pm 3.0$ & $66.4 \pm 6.1^{*}$ & $83.2 \pm 3.0$ & $69.5 \pm 11.0^{*}$ & $58.4 \pm 7.1^{* *}$ \\
Acrosome reaction (\%) & $22.3 \pm 1.2$ & $9.3 \pm 0.7^{* * *}$ & $19.3 \pm 1.8$ & $8.8^{* * *}$ & $12.0 \pm 1.2^{* * *}$ \\
$\%$ PUFA/control & 100 & $79.1 \pm 5.8^{*}$ & $99.0 \pm 0.9$ & $96.5 \pm 4.9$ & $81.5 \pm 6.3^{*}$ \\
\hline
\end{tabular}

Values are means \pm SEM, $n=5$.

$* P<0.01 ; * * P<0.005 ; * * * P<0.001$ compared with control values. 
Table 3. Influence of the combination of xanthine $\left(X ; 200 \mu \mathrm{mol} \mathrm{l} \mathrm{l}^{-1}\right)$ and xanthine oxidase $\left(X \mathrm{O} ; 50 \mathrm{mU} \mathrm{ml}^{-1}\right), \mathrm{H}_{2} \mathrm{O}_{2}$ and mercaptosuccinate (MCS) on the superoxide dismutase (SOD)-like and glutathione peroxidase/reductase (GPX/GRD) activities, oxidized glutathione/reduced glutathione (GSSG/GSH) content, and hydroperoxide concentrations after $3 \mathrm{~h}$ of incubation

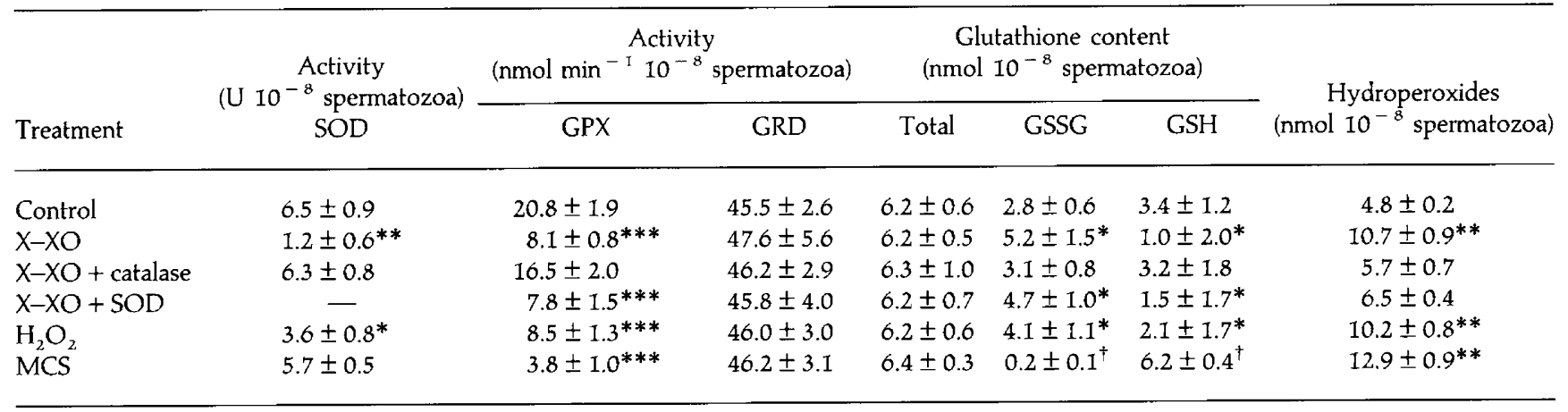

Values are means $\pm \mathrm{SEM}, n=7$.

GSH was calculated as the difference between the total glutathione content and GSSG.

Statistical analyses were performed using the unpaired Student's $t$-test.

$* P<0.05 ; * * p<0.01 ; * * * P<0.001$ compared with control values, ${ }^{\dagger} P<0.01$ compared with all other groups.

Table 4. Protective effects of histidine on hydroperoxides and membrane polyunsaturated fatty acid (PUFA) content after treatment by the xanthine $\left(X ; 200 \mu \mathrm{mol} \mathrm{l}^{-1}\right)$ and xanthine oxidase $\left(X \mathrm{O} ; 50 \mathrm{mU} \mathrm{ml}^{-1}\right)$

\begin{tabular}{lcc}
\hline Treatment & $\begin{array}{c}\text { Hydroperoxides } \\
\text { (nmol } 10^{-8} \text { spermatozoa) }\end{array}$ & \% PUFA/control \\
\hline Control & $5.5 \pm 0.5$ & 100 \\
X-XO & $10.2 \pm 0.8^{* * *}$ & $79.1 \pm 5.8^{* *}$ \\
$X-X O+$ histidine & $7.6 \pm 0.5^{*}$ & $92.5 \pm 1.4^{*}$ \\
\hline
\end{tabular}

Values are means \pm SEM, $n=5$.

${ }^{*} P<0.05 ;{ }^{* * P}<0.01 ;{ }^{* * *} P<0.005$ compared with control values.

G6PDH is known to be very susceptible to oxidative stress (Oliver et al., 1987a), and its inactivation may lead to the blockade of the pentose phosphate pathway, and a resulting decrease in NADPH concentration in the cell, leading to a decrease in the antioxidative protection exerted by the glutathione-dependent system. This has been shown to occur in other biological models (Starke-Reed and Oliver, 1989) and also in physiological processes like ageing (Noy et al., 1985; Oliver $e t$ al., 1987b) and the hypothesis can now be extended to spermatozoa. The activity of G6PDH is partially impaired by the xanthine-xanthine oxidase system. This significant decrease of the G6PDH activity may be one factor leading to a decrease of NADPH in spermatozoa and the inhibition of the recycling of GSSG into GSH despite the unaltered enzymatic activity of the glutathione reductase. In recent studies, De Lamirande and Gagnon (1992a, b) showed that reactive oxygen species produced by xanthine and xanthine oxidase, or by $\mathrm{H}_{2} \mathrm{O}_{2}$ interfere with the energy production metabolism in human spermatozoa leading to a drop in ATP concentrations. They hypothesized that these effects may be due to the inhibition of glyceraldehyde-3-phosphate dehydrogenase, a key enzyme of the glycolytic pathway. The results presented in this study show that reactive oxygen species can effectively inhibit a metabolic pathway involved in energy production
(NADPH). They also suggest that such an inhibition can have important consequences for the antioxidant protection of spermatozoa, and point out a possible link between these two phenomena.

The decrease of the superoxide dismutase-like and glutathione peroxidase activities after addition of $\mathrm{H}_{2} \mathrm{O}_{2}$ suggests that the mechanism by which $\mathrm{H}_{2} \mathrm{O}_{2}$ mediates its effects on spermatozoa may be linked to the inhibition of the activities of these two protective enzymes. However, the activity of glutathione reductase remained unchanged pointing out that the observed reduction of superoxide dismutase-like and glutathione peroxidase activities were not due to leakage of the enzymes from peroxidized cells. It has been shown that the decrease of glutathione peroxidase activity induced by the xanthine-xanthine oxidase system or by $\mathrm{H}_{2} \mathrm{O}_{2}$ was linked to a direct inhibition of glutathione peroxidase. Such an inhibition by hydroperoxides has been described in mammalian cells and shown to be associated to an oxidative modification of the protein (Ochi, 1990). The selenium atoms present at the catalytic site of the enzyme undergo a redox cycle during the enzymatic detoxification of hydroperoxides by glutathione peroxidase, between a reduced (selenol) and an oxidized form (selenic acid) (Flohe, 1982 cited in Ochi, 1990). It was hypothesized that the increase of the oxidation level of the cell slows down the return to the reduced state of selenium, leading to a reversible inhibition of the enzyme. Thus, if the observed oxidation of GSH to GSSG does not account directly for the decreased glutathione peroxidase activity in our model, the depletion of reducing substances in the spermatozoa after xanthine-xanthine oxidase treatment may be responsible for the presence of the inactive form of the enzyme.

$\mathrm{H}_{2} \mathrm{O}_{2}$-induced impairment of superoxide dismutase activity has been previously reported (Salo et al., 1990; Zini et al., 1993) but it is the first time, to our knowledge, that it is shown to occur in human spermatozoa after reactive oxygen species treatment. Superoxide dismutase plays a key role in protecting human spermatozoa since its activity is well correlated with time of motility loss (Alvarez et al., 1987). The inhibition of superoxide dismutase-like activity by $\mathrm{H}_{2} \mathrm{O}_{2}$ and the 

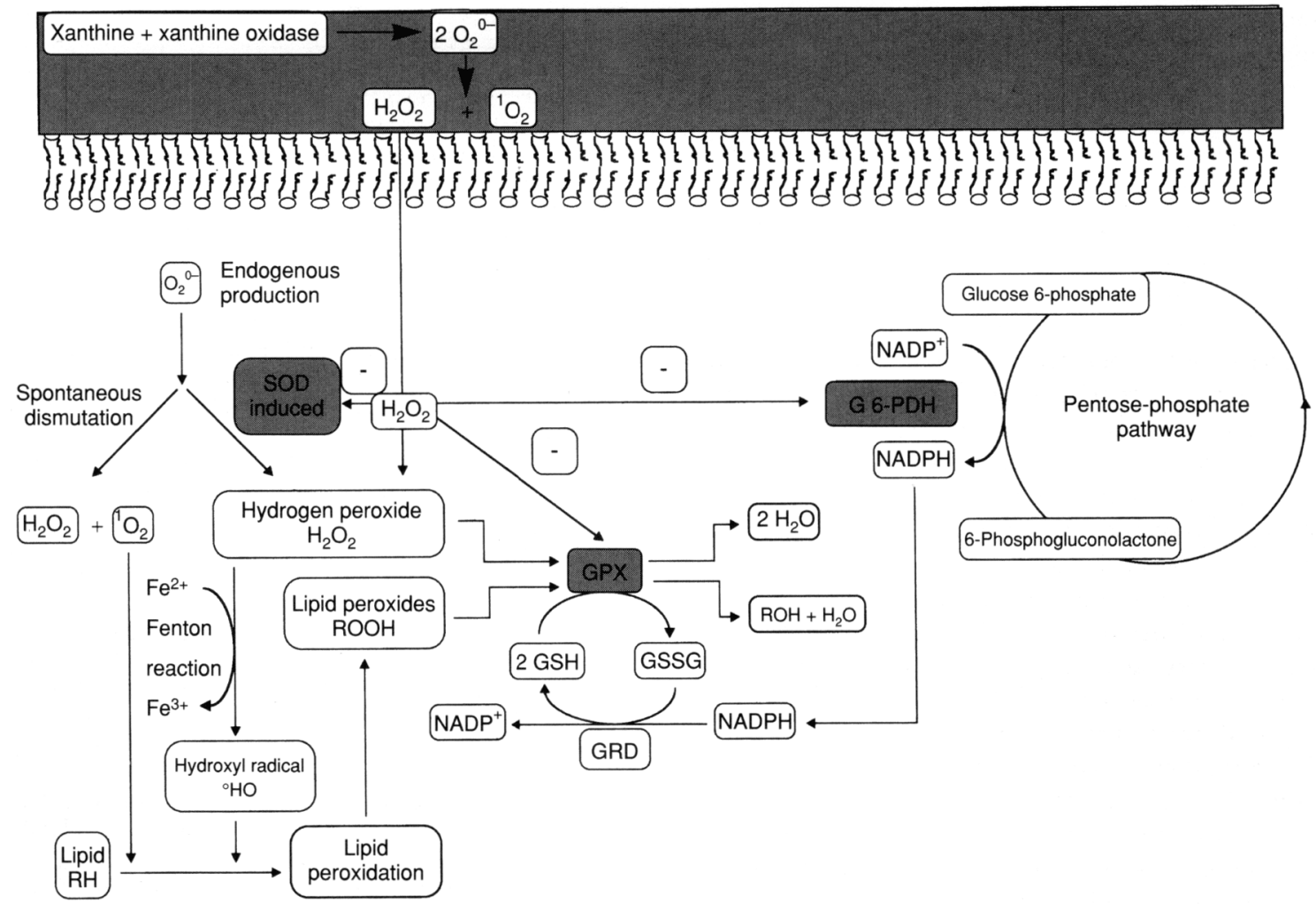

Fig. 4. Schematic representation for the hypothesis of the events triggered by the xanthine-xanthine oxidase system on some enzyme activities of human spermatozoa. Lipid peroxidation is shown as the result of the hydrogen peroxide-induced inhibition of the antioxidant enzymatic defence systems of the spermatozoa. By decreasing the superoxide dismutase, glutathione peroxidase and glucose-6-phosphate dehydrogenase activities, hydrogen peroxide would permit, in synergy with the endogenous production of reactive oxygen species, the accumulation of toxic lipid peroxides and the development of lipid peroxidation. SOD: superoxide dismutase; GPX: glutathione peroxidase; GRD: glutathione reductase; G6PDH: glucose-6-phosphate dehydrogenase; GSSG: oxidized glutathione; GSH: reduced glutathione.

consequences of decreased glutathione peroxidase activity on the functional properties of human spermatozoa emphasize the importance of these two enzymes in the antioxidant defence system of spermatozoa, and the importance of their cooperation in exerting optimal protection of the cells. The role of glutathione peroxidase in the antioxidant protection of the lipid constituents of spermatozoa is further shown by its direct inactivation by mercaptosuccinate, which provokes a rise in the concentration of lipid hydroperoxides.

Lipid peroxidation was induced by the xanthine-xanthine oxidase system, as shown by the increase in the lipid hydroperoxide content and the degradation of membrane PUFAs. $\mathrm{H}_{2} \mathrm{O}_{2}$ and $\mathrm{O}_{2}{ }^{\circ-}$ were both involved in the initiation of peroxidation since catalase and superoxide dismutase can prevent the degradation of the PUFAs. It is known that $\mathrm{H}_{2} \mathrm{O}_{2}$ and $\mathrm{O}_{2}{ }^{\circ-}$ can produce ${ }^{\circ} \mathrm{OH}$ in the presence of transition metals (Fenton reaction), but as Aitken et al. (1993a) reported, mannitol was ineffective in protecting spermatozoa against xanthine-xanthine oxidase-induced lipoperoxidation. However, the involvement of ${ }^{\circ} \mathrm{OH}$ radicals cannot be ruled out. Mannitol is a polar molecule restricted to the extracellular medium, whereas $\mathrm{H}_{2} \mathrm{O}_{2}$ can diffuse freely across biological membranes. An intracellular Fenton reaction is possible with ${ }^{\circ} \mathrm{OH}$ scavengers and iron chelators capable of penetrating cells. This possibility is emphasized by results showing that dimethyl sulfoxide, a lipophilic ${ }^{\circ} \mathrm{OH}$ scavenger, delays the immobilization of spermatozoa induced by a xanthinexanthine oxidase system (De Lamirande and Gagnon, 1992a). Moreover, the cellular functions inhibited by $\mathrm{H}_{2} \mathrm{O}_{2}$, the acrosome reaction and motility are dependent on intracellular structures such as acrosomes, mitochondria and axonemes. As $\mathrm{H}_{2} \mathrm{O}_{2}$ is produced in the extracellular medium by the xanthine-xanthine oxidase system, this may imply that $\mathrm{H}_{2} \mathrm{O}_{2}$ must cross the plasma membrane to exert its toxic effects. Singlet oxygen reacts strongly with unsaturated fatty acids to form hydroperoxides (Rawls and Van Santen, 1970). Histidine is known to be an ${ }^{1} \mathrm{O}_{2}$ scavenger (Lynch and Fridovich, 1978) but can also react with ${ }^{\circ} \mathrm{OH}$. The inefficacy of mannitol to protect the spermatozoa, the conversion of 1,3 diphenylisobenzofuran to $o$-dibenzoylbenzene in the presence of the xanthine-xanthine oxidase system and the partial protection afforded to the PUFAs by histidine suggest that ${ }^{\mathrm{I}} \mathrm{O}_{2}$ oxidation of fatty acids significantly contributes to the lipid alterations of spermatozoa induced by the xanthinexanthine oxidase system.

The results presented in this study lead us to propose the following sequence of events triggered by the xanthinexanthine oxidase system against human spermatozoa (Fig. 4). First, the xanthine-xanthine oxidase system produces $\mathrm{O}_{2}{ }^{\circ}$ which dismutates spontaneously into $\mathrm{H}_{2} \mathrm{O}_{2}$ and possibly ${ }^{1} \mathrm{O}_{2}$. 
Hydrogen peroxide diffuses across the membranes into the cells and inhibits the activities of some enzymes: G6PDH, leading to a decrease in the production of NADPH and a concomitant accumulation of GSSG, glutathione peroxidase and superoxide dismutase, leading to a decrease in the antioxidant defences of the spermatozoa. The disorganization of the antioxidant defences then leads to the peroxidation of the membrane phospholipids by reactive oxygen species. As a consequence of the changes in membrane fluidity and integrity induced by the accumulation of lipid peroxides, the ionophoreinduced acrosome reaction and sperm movement are disrupted.

Much remains to be directly studied, but this hypothesis takes into account the results obtained in the many studies on reactive oxygen species and human spermatozoa. $\mathrm{H}_{2} \mathrm{O}_{2}$ appears to be the most toxic species because of its ability to cross membranes freely and to inhibit enzyme activities and cellular functions. The inhibition of energy metabolism by $\mathrm{H}_{2} \mathrm{O}_{2}$ may then explain the reversible immobilization of spermatozoa. Overall membrane degradation, as measured by the phospholipid fatty acids degradation, appears to occur later, possibly as a consequence of the inhibition of G6PDH, glutathione peroxidase and superoxide dismutase activities. A global lipid peroxidation may indicate a switch between reversible alterations due to the early inhibition of energy metabolism and irreversible structural degradations. $\mathrm{H}_{2} \mathrm{O}_{2}$ produced by xanthine and xanthine oxidase is responsible for the inhibition of the antioxidant defence system of spermatozoa, but the production of reactive oxygen species by this pro-oxidant system stops rapidly. This suggests that the reactive oxygen species responsible for the lipid alterations may be produced by the spermatozoa themselves. It also suggests that reactive oxygen species induce chain reactions independent of the reactive oxygen species themselves, the effects of which are observed only hours later.

\section{References}

Aitken RJ and Clarkson JS (1987) Cellular basis of defective sperm function and its association with the genesis of reactive oxygen species by human spermatozoa Joumal of Reproduction and Fertility 81 459-469

Aitken RJ and West KM (1990) Analysis of the relationship between reactive oxygen species production and leucocyte infiltration in fractions of human semen separated on Percoll gradients International Journal of Andrology 13 433-451

Aitken RJ, Clarkson IS and Fishel S (1989) Generation of reactive oxygen species, lipid peroxidation and human sperm function Biology of Reproduction 40 183-197

Aitken RJ, Irvine DS and Wu FC (1991) Prospective analysis of sperm-cocyte fusion and reactive oxygen species generation as criteria for the diagnosis of infertility American Journal of Obstetrics and Gynecology 164 542-551

Aitken RJ, Buckingham D, West K, Wu FC, Zikopoulos K and Richardson DW (1992a) Differential contribution of leucocytes and spermatozoa to the high levels of reactive oxygen species recorded in the ejaculates of oligozoospermic patients Journal of Reproduction and Fertility 94 $451-462$

Aitken RJ, Buckingham DW and West KM (1992b) Reactive oxygen species and human spermatozoa, analysis of the cellular mechanisms involved in luminoland lucigenin-dependent chemiluminescence Journal of Cellular Physiology 151 466-477

Aitken RJ, Buckingham DW and Harkiss D (1993a) Use of a xanthine oxidase free radical generating system to investigate the cytotoxic effects of reactive oxygen species on human spermatozoa Journal of Reproduction and Fertility $97441-450$

Aitken RJ, Harkiss D and Buckingham DW (1993b) Relationship between iron-catalysed lipid peroxidation potential and human sperm function Journal of Reproduction and Fertility 98 257-265

Alvarez JG and Storey BT (1983) Role of superoxide dismutase in protecting rabbit spermatozoa from $\mathrm{O}_{2}$ toxicity due to lipid peroxidation Biology of Reproduction 28 1129-1136

Alvarez JG and Storey BT (1989) Role of glutathione peroxidase in protecting mammalian spermatozoa from loss of motility caused by spontaneous lipid peroxidation Gamete Research 23 77-90

Alvarez JG, Touchstone JC, Blasco I and Storey BT (1987) Spontaneous lipid peroxidation and production of hydrogen peroxide and superoxide in human spermatozoa Journal of Andrology 8 338-348

Burkman LJ (1991) Discrimination between nonhyperactivated and classical hyperactivated motility patterns in human spermatozoa using computerized analysis Fertility and Sterility $\mathbf{5 5} 363-371$

Comporti M (1989) Three models of free radical induced cell injury Chemical Biological Interactions 72 1-56

Corey EJ, Mehrotra MM and Khan AU (1987) Water-induced dismutation of superoxide anion generates singlet molecular oxygen Biochemical and Biophysical Research Communication 145 842-846

De Lamirande E and Gagnon C (1992a) Reactive oxygen species and human spermatozoa I. Effects on the motility of intact spermatozoa and on sperm axonemes Journal of Andrology 13 368-378

De Lamirande E and Gagnon C (1992b) Reactive oxygen species and human spermatozoa II. Depletion of adenosine triphosphate plays an important role in the inhibition of sperm motility Journal of Andrology 13 379-386

Folch J, Lees M and Sloane-Stanley GH (1957) A simple method for isolation and purification of total lipids from animal tissues Joumal of Biological Chemistry 226 497-502

Gavella M, Lipovac V and Marotti T (1991) Effect of pentoxifylline on superoxide anion production by human sperm International Journal of Andrology 14 320-327

Hagenfeld L (1966) A gas chromatographic method for the determination of fatty acids in plasma Clinica et Chimica Acta 13 266-268

Hicks M and Gebicki JM (1979) A spectrophotometric method for the determination of lipid hydroperoxides Analytical Biochemistry 99 249-253

Hindshaw DB, Sklar LA and Bohl B (1986) Cytoskeletal and morphologic impact of cellular oxidant injury American Journal of Pathology 123 454-464

Iwasaki A and Gagnon C (1992) Formation of reactive oxygen species in spermatozoa of infertile patients Fertility and Sterility 57 409-416

Jeulin C, Soufir JC, Weber P, Laval-Martin D and Calvayrac R (1989) Catalase activity in human spermatozoa and seminal plasma Gamete Research 24 $185-196$

Jones R, Mann T and Sherins RJ (1979) Peroxidative breakdown of phospholipids in human spermatozoa: spermicidal effects of fatty acid peroxides and protective action of seminal plasma Fertility and Sterility 31 531-537

Kellogg EW III and Fridovich I (1975) Superoxide, hydrogen peroxide, and singlet oxygen in lipid peroxidation by a xanthine oxidase system Journal of Biological Chemistry $2508812-8817$

Kellogg EW III and Fridovich I (1977) Liposome oxidation and erythrocyte lysis by enzymically generated superoxide and hydrogen peroxide Journal of Biological Chemistry 25 6721-6728

Kessopoulou E, Tomlinson MJ, Barratt CLR, Bolton AE and Cooke ID (1992) Origin of reactive oxygen species in semen; spermatozoa or leukocytes? Journal of Reproduction and Fertility 94 463-470

Kobayashi T, Miyazaki T, Natori M and Nozawa S (1991) Protective role of superoxide dismutase in human sperm motility: superoxide dismutase activity and lipid peroxide in human seminal plasma and spermatozoa Human Reproduction 6 987-991

Koppenol WH (1976) Reactions involving singlet oxygen and the superoxide anion Nature 262 420-421

Le Lannou D, Griveau JF, Le Pichon JP and Quero JC (1992) Effects of chamber depth on the motion pattern of human spermatozoa in semen or in capacitating medium Human Reproduction 7 1417-1421

Lynch RE and Fridovich I (1978) Effects of superoxide on the erythrocyte membrane Journal of Biological Chemistry 253 1838-1845

Mortimer ST and Mortimer D (1990) Kinematics of human spermatozoa incubated under capacitating conditions Joumal of Andrology 11 195-203

Nakano M, Takayama K, Shimizu Y, Tsuji Y, Inaba H and Migita T (1976) Spectroscopic evidence for the generation of singlet oxygen in self-reaction of sec-peroxy radicals Journal of American Chemical Society 98 1974-1975

Nissen HP and Kreysel HW (1983) Superoxide dismutase in human semen Klinische Wochenschrift $\mathbf{6 1} 63-65$ 
Noy N, Schwartz H and Gafni A (1985) Age-related changes in the redox status of rat muscle cells and their role in enzyme-ageing Mechanisms of Ageing and Development $2963-69$

Ochi T (1990) Effects of an organic hydroperoxide on the activity of antioxidant enzymes in cultured mammalian cells Toxicology 61 229-239

Oliver CN, Ahn BW, Moerman EJ, Goldstein S and Stadtman ER (1987a) Age-related changes in oxidized proteins Journal of Biological Chemistry 262 5488-5491

Oliver CN, Levine RL and Stadtman ER (1987b) A role of mixed-function oxidation reactions in the accumulation of altered enzyme forms during aging Journal of American Geriatric Society 35 947-956

Pederson TC and Aust SD (1973) The role of superoxide and singlet oxygen in lipid peroxidation promoted by xanthine oxidase Biochemical and Biophysical Research Communications 52 1071-1078
Rawls HR and Van Santen PJ (1970) Singlet oxygen: a possible source of the original hydroperoxides in fatty acids Journal of American Oil Chemical Society 171 135-138

Salo DC, Pacifici RE, Lin SW, Giulivi C and Davies KJ (1990) A superoxide dismutase undergoes proteolysis and fragmentation following oxidative modification and inactivation Joumal of Biological Chemistry 265 11919-11927

Starke-Reed PE and Oliver CN (1989) Protein oxidation and proteolysis during aging and oxidative stress Archives of Biochemistry and Biophysics 275 559-567

Zini A, De Lamirande E and Gagnon C (1993) Reactive oxygen species in semen of infertile patients: levels of superoxide dismutase- and catalaselike activities in seminal plasma and spermatozoa International journal of Andrology 16 183-188 